\title{
Essential Fatty Acid Deficiency Ameliorates Acute Renal Dysfunction in the Rat after the Administration of the Aminonucleoside of Puromycin
}

\author{
K. P. G. Harris, ${ }^{\star}$ J. B. Lefkowith, ${ }^{\ddagger}$ S. Klahr, ${ }^{*}$ and G. F. Schreiner ${ }^{\star 5}$ \\ ${ }^{*}$ Renal Division, Department of Medicine, and the Departments of ${ }^{\ddagger}$ Pharmacology and ${ }^{\S}$ Pathology, \\ Washington University School of Medicine, St. Louis, Missouri 63110
}

\begin{abstract}
The administration of the aminonucleoside of puromycin (PAN) to rats causes the nephrotic syndrome that is associated with an acute decline in renal function, and an interstitial infiltrate. We examined whether essential fatty acid deficiency (EFAD), which inhibits macrophage infiltration in glomerulonephritis, affects PAN-induced renal dysfunction.

Both control and EFAD rats developed proteinuria that resolved over 28 d. After PAN administration, there was a prominent infiltration of macrophages in rats fed a normal diet. The infiltrate was prevented by the EFAD diet. The absence of a macrophage interstitial infiltrate was associated with a significantly higher $C_{i n}$ in the EFAD rats than in controls at $7 \mathrm{~d}$ $(5.21 \pm 1.19$ versus $0.39 \pm 0.08, P<0.002 \mathrm{ml} / \mathrm{min} / \mathrm{kg} \mathrm{BW})$. In addition, $C_{P A H}$ fell to $<10 \mathrm{ml} / \mathrm{min} / \mathrm{kg} \mathrm{BW}$ by day 7 in controls, but remained the same as normal in the EFAD. After administration of PAN to control rats, there was no increase in urinary thromboxane excretion or an increase in glomerular thromboxane production. Furthermore, the effect of EFAD could not be mimicked by the administration of a thromboxane synthase inhibitor. Irradiation-induced leukopenia in rats on a normal diet markedly improved glomerular filtration and renal blood flow in acutely nephrotic rats.

EFAD prevents the interstitial cellular infiltrate and the renal ischemia associated with experimental nephrosis. The recruitment of mononuclear cells into the kidney following PAN directly contributes to the decline in renal function. ( $J$. Clin. Invest. 1990. 86:1115-1123.) Key words: essential fatty acids • macrophages $\bullet$ nephrosis $\bullet$ azotemia
\end{abstract}

\section{Introduction}

After a single injection of the aminonucleoside of puromycin $(\mathrm{PAN})^{1}$ rats develop heavy proteinuria, which resolves after 4-5 wk (1-3). Aminonucleoside nephrosis has been widely used as a model of human minimal change disease, with which it was thought to share similar ultrastructural changes $(1,4)$. However, even initial reports of this model recognized that the development of the nephrotic syndrome was associated with azotemia (1), and subsequent studies have confirmed that there is a decline in both whole kidney and single nephron glomerular filtration rate $(5,6)$. Renal plasma flow and glo-

Received for publication 7 June 1989 and in revised form 21 May 1990.

1. Abbreviations used in this paper: BUN, blood urea nitrogen; BW, body weight; EFAD, essential fatty acid deficiency; PAH, P-aminohippurate; PAN, aminonucleoside of puromycin.

J. Clin. Invest.

(c) The American Society for Clinical Investigation, Inc.

0021-9738/90/10/1115/09 \$2.00

Volume 86, October 1990, 1115-1123 merular capillary plasma flow are also reduced (2) as is the ultrafiltration coefficient (6). The etiology of the acute decrease in glomerular filtration rate remains ill understood in this model. Administration of PAN also modulates the number and state of activation of glomerular macrophages. The first week is characterized by no change in glomerular macrophage number, and the second week by a moderate glomerular infiltration by monocytes and an increase in Ia expression by both resident and infiltrating cells (7). In addition, Eddy and Michael (2) recently described the development of an acute reversible interstitial nephritis following the administration of $15 \mathrm{mg} / 100 \mathrm{~g} \mathrm{BW}$ of PAN, consisting predominantly of macrophages.

Modification of dietary fatty acids has been shown to prolong survival in several animal models of systemic lupus erythematosus, probably by preventing the glomerulonephritis which is the principal cause of mortality $(8,9)$. An experimental basis for this protective effect has now been provided by the observations that, when rats are reared on a diet deficient in essential fatty acids, there is depletion of resident glomerular macrophages and inhibition of angiotensin II-induced glomerular eicoisanoid production (10). Furthermore, animals that are deficient in essential fatty acids do not infiltrate their glomeruli with macrophages in response to a variety of immune stimuli $(11,12)$. To date, however, no quantitative data exists on whether these animals develop an interstitial infiltrate in response to appropriate stimuli, although basal levels of interstitial macrophages are reduced (10).

We therefore examined whether rats raised on a diet deficient in essential fatty acids would be protected from the development of an interstitial infiltrate in response to PAN, and whether this would convey any benefit in terms of preventing the renal functional changes.

The generation of the vasoconstrictor thromboxane $A_{2}$ has been implicated in the pathogenesis of the nephrotic syndrome induced by the administration of adriamycin (13). Since essential fatty acid deficient (EFAD) animals have a decreased ability to synthesize the vasoconstrictor thromboxane $A_{2}$, we further investigated the role of thromboxane $A_{2}$ in the development of PAN nephrosis, both by measuring its production and by studying the effect of the thromboxane synthase inhibitor OKY-046 on the development of renal failure after the administration of PAN.

\section{Methods}

Female Lewis rats (Harlan Sprague Dawley, Indianapolis, IN) were used in all experiments. Age-matched animals were fed either a standard rat chow or a diet deficient in essential fatty acids (10) (Ralston Purina, St. Louis, MO) from the time of weaning. The animals were maintained on the diet for at least 4 mo before use in the experiments, at which time they weighed between 175 and $250 \mathrm{~g}$. The diets were matched for protein $(22 \%)$. 
To determine the time course of the changes in renal function after the administration of PAN, 11 animals (5 control and 6 EFAD) were given $15 \mathrm{mg} / 100 \mathrm{~g}$ BW of PAN (ICN Biochemicals, Cleveland, $\mathrm{OH}$ ) by intraperitoneal injection after the collection of a basal $24 \mathrm{~h}$ urine and a fasting tail vein blood sample. Urine was assayed for protein and thromboxane $B_{2}$ and plasma was analyzed for blood urea nitrogen (BUN), plasma creatinine, albumin, cholesterol, and triglyceride. Further 24-h urine collections and blood samples were obtained 7, 14, 21, and $28 \mathrm{~d}$ after the administration of PAN.

In a separate group of animals, standard renal clearance studies were performed as described below. The animals were studied after the collection of a 24-h urine, at $4 \mathrm{~d}$ (control, $n=5$; EFAD, $n=4$ ) and $7 \mathrm{~d}$ (control, $n=6$; EFAD, $n=6$ ) after the administration of $15 \mathrm{mg} / \mathrm{kg} \mathrm{BW}$ of PAN. These animals were pair fed $1 \mathrm{wk}$ before the administration of PAN up to the time of study. Clearance studies were also performed in an additional nine rats that had not received PAN (control, $n=5$; EFAD, $n=4$ ). The data obtained from analysis of plasma for BUN, plasma creatinine, albumin, cholesterol, triglyceride, and urine for protein in these animals was pooled with that obtained at day 7 in the animals studied to determine the time course of changes after PAN administration.

Studies with the thromboxane synthase inhibitor OKY-046. To examine the effect of inhibiting the generation of the vasoconstrictor thromboxane $A_{2}$ on the development of the renal impairment in this model rats were treated for $7 \mathrm{~d}$ after the administration of PAN with the inhibitor of thromboxane synthesis OKY-046 (Ono Pharmaceuticals, Osaka, Japan). The OKY-046 at a dose of $20 \mathrm{mg} / \mathrm{kg} \mathrm{BW}$ was administered subcutaneously twice daily to six rats on the control diet. We have reported previously that this dose and route of administration of OKY-046 or one of its analogues is effective in affecting renal function and inhibiting thromboxane excretion in the urine $(14,36)$. An additional three rats from the control diet group were given PAN, and only saline instead of OKY-046 to serve as temporal controls. A group $(n=5)$ of littermates receiving neither PAN nor injections served as additional controls. Standard renal clearances were performed $7 \mathrm{~d}$ after the administration of PAN as described below.

Clearance studies. Animals were prepared for standard renal clearance measurements in the awake state. Tail vein, femoral artery, and urinary bladder catheters were inserted under ether anesthesia, and the animal was secured in a plexiglass holder and allowed to recover from anesthesia for at least $1 \mathrm{~h} .1 \mathrm{~h}$ before study a priming dose of inulin (Fisher Scientific Co., St. Louis, MO) designed to give plasma levels of 75-125 mg/dliter and P-aminohippurate (PAH) (Merck, Sharp, and Dohme, West Point, PA) designed to give plasma levels of 0.75-1.5 $\mathrm{mg} / \mathrm{dl}$ were given via the tail vein. This was followed by a sustained infusion containing inulin $(32 \mathrm{mg} / \mathrm{ml})$ and PAH $(1.6 \mathrm{mg} / \mathrm{ml})$ given at $40 \mu \mathrm{l} / \mathrm{min}$ to maintain constant plasma levels (for the rats studied $7 \mathrm{~d}$ after the administration of PAN, the concentrations of inulin and PAH in the sustain infusion was halved to allow for the decreased glomerular filtration rate). After $1 \mathrm{~h}$ of equilibration, two consecutive 20-min urine collections, with blood samples obtained at the midportion of each period were made for calculation of whole kidney inulin and $\mathrm{PAH}$ clearances $\left(C_{\text {in }}\right.$ and $\left.C_{P A H}\right)$. At the end of the experiment, the animal was killed by rapid injection of pentobarbital, and one kidney was removed. One half of the kidney was snap frozen flat in dry ice and ethanol for subsequent immunoperoxidase labeling.

Studies utilizing bone marrow irradiation. To evaluate the effects of leukocyte depletion, one group of rats received whole body irradiation of 1,315 rads over 10 min (Gammacell 40; Atomic Energy of Canada, Ltd., Ottowa, Canada). A lead cuff of 0.5-cm thickness was placed to shield the kidneys anteriorly and posteriorly; we have shown this procedure to block $>92 \%$ of the irradiation in the immediate vicinity of the kidneys (15). The irradiation was delivered $4 \mathrm{~d}$ after the administration of PAN and $3 \mathrm{~d}$ before sacrifice for the purpose of leukocyte labeling and clearance studies. This is a modification of a previously published protocol that we have employed to deplete the kidney of resident interstitial leukocytes and to inhibit the influx of leukocytes into the cortex in acute ureteral obstruction $(15,16)$.
Immunoperoxidase labeling of renal tissue. The interstitial macrophages were enumerated by labeling kidney sections with a mouse monoclonal antibody directed against the ED1 antigen, a cytoplasmic antigen of rat monocytes and tissue macrophages (17). Frozen sections $(6 \mu \mathrm{m})$ of the kidneys were fixed in chilled $\left(4^{\circ} \mathrm{C}\right)$ acetone for $10 \mathrm{~min}$ and air dried. Endogenous peroxidase activity was inhibited by immersion for $10 \mathrm{~min}$ in a solution consisting of $\mathbf{4 0}$ parts methanol, 9 parts water, and 1 part hydrogen peroxide (30\%). Endogenous biotin activity was inhibited by sequential 30 -min exposures to Avidin $D$ and Biotin blocking solutions (Vector Laboratories, Burlingame, CA). The sections were then placed in horse serum $(10 \%)$ blocking solution (Zymed Laboratories, San Francisco, CA), followed by ED-1 antimacrophage antibody (Accurate Chemical \& Scientific Corp., Westbury, NY) (diluted 1:10 in PBS containing 3.5\% BSA) for $30 \mathrm{~min}$. After washing, biotinylated horse anti-mouse IgG (Vector Laboratories) affinity purified and adsorbed against rat IgG, diluted 1:50, was added for $15 \mathrm{~min}$. After washing, the slides were sequentially exposed to streptavidin-peroxidase followed by aminoethyl carbazole and hydrogen peroxide, as supplied by Zymed Laboratories. The sections were counterstained with hematoxylin and mounted under coverslips.

Macrophage number was quantified as the number of cells per high power field $(450 \times) .15$ fields were counted in each animal, and a mean was taken.

Glomerular macrophage labeling. Glomeruli were labeled for macrophages in three rats (normal diet) $7 \mathbf{d}$ after the administration of PAN and in two noninjected rats, using a protocol modified from that previously published (17). The kidneys were perfused with PBS at $37^{\circ} \mathrm{C}$ to which was added papaverine at a concentration of $100 \mu \mathrm{g} / \mathrm{ml}$ to eliminate the contribution of circulating leukocytes to the enumeration of the cells in the glomerulus. The cortex was dissected from the medulla and passed through sieves of graded sizes to separate the glomeruli. The glomeruli were then placed in a digestion medium consisting of HBSS, containing 1\% Hepes buffer, Type 2 collagenase $(500 \mu \mathrm{g} / \mathrm{ml})$, DNase $(0.01 \mathrm{mg} / \mathrm{ml})$, and soybean trypsin inhibitor $(1$ $\mathrm{mg} / \mathrm{ml}$ ). The glomeruli were rotated in this mixture at room temperature for $\mathbf{3 0} \mathrm{min}$, washed twice, and then placed in PBS containing polyvalent rabbit anti-rat macrophage antiserum absorbed against other rat leukocytes (Accurate Chemical Scientific Corp.), at a concentration of $50 \mu \mathrm{g} / \mathrm{ml}$, to label resident glomerular macrophages. After $20 \mathrm{~min}$ at $4^{\circ} \mathrm{C}$, the glomeruli were washed once, placed in PBS for $10 \mathrm{~min}$ to permit unbound antibody to diffuse out of the glomerulus, washed again, and placed in a solution of fluorescein-conjugated goat anti-rabbit IgG (Accurate Chemical Scientific Corp.) at a concentration of $100 \mathrm{mg} / \mathrm{ml}$ in PBS. This technique has previously been shown to render intact glomeruli permeable to antibody, allowing in situ cells to be labeled (18). The labeled cell content of isolated glomeruli was evaluated by microscopic examination with a Zeiss universal microscope. Cells were quantified by focusing through the glomeruli and counting cells as they appeared in the plane of focus. Because exposure to the enzymes is limited, overall glomerular architecture is not disturbed, permitting accurate evaluation of the macrophage content of glomeruli on a per glomerulus basis. The glomeruli from the three PAN treated animals were pooled, as were those from the controls, and the macrophage number of $\mathbf{4 0}$ glomeruli were counted.

Quantitation of the leukocyte content of the cortex. In some experiments, the leukocyte content of the renal cortex was determined by the enzymatic digestion of the perfused renal cortex to a suspension of dissociated cells, followed by the immunofluorescent labeling of the cells for the leukocyte common antigen. Details of this procedure have been previously presented (19). Leukocyte content was determined for control kidneys, kidneys on day 7 after the administration of PAN, and kidneys from animals that had been irradiated $4 \mathrm{~d}$ after the administration of PAN and $3 \mathrm{~d}$ before killing the animals. Leukocyte content was expressed as the number of cells expressing the leukocyte common antigen per gram wet weight of renal cortex.

Glomerular eicosanoid production. Glomeruli were isolated from saline-perfused kidneys from six rats fed the control diet $7 \mathrm{~d}$ after the administration of PAN and from six nonnephrotic rats using a pre- 
viously detailed sieving protocol (18). To obtain sufficient material for the prostanoid assays, kidneys from two rats were pooled. Preparations were typically $>90 \%$ pure. Glomeruli were subsequently permeabilized with collagenase and DNase as described previously. This treatment is necessary to preserve glomerular viability and responses to agonists in vitro (10).

Glomeruli were incubated in Kreb's-Henseleit buffer $(\sim 30,000$ glomeruli/0.4 ml) for sequential 10-min periods. After two basal periods, glomeruli were washed three times and a basal period of eicosanoid production was performed. Glomeruli were subsequently exposed to ionomycin $10 \mu \mathrm{M}$ and eicosanoid production was again measured.

Glomerular supernatants were then assayed for $\mathrm{PGE}_{2}, \mathrm{TxB}_{2}$, and $\mathrm{LTB}_{4}$ content by specific radioimmunoassays detailed before (20). The values were normalized for the number of glomeruli in the incubation and are expressed as $\mathrm{fmol} / 10^{3}$ glomeruli.

Hepatic lipid analysis. Hepatic fatty acid composition was periodically monitored in the groups of animals used in the study to confirm that the animals on the EFAD diet were indeed essential fatty acid deficient. Livers from animals fed the control and EFAD diets were extracted using a Bligh-Dyer extraction (21). Lipids were then transmethylated, and the resultant fatty acid methyl esters were isolated by thin layer chromatography and characterized by gas chromatography using previously described methods (22).

Analytic. Inulin in plasma and urine was determined using the method of Fuhr (23), and PAH was measured by a modification of the method of Smith et al. (24). Sodium was measured by standard flame photometry (Instrumentation Laboratory, Inc., Lexington, MA). Urinary urea, BUN, creatinine, triglyceride, cholesterol, and albumin in plasma were measured using colorimetric methods on a Multistat III plus autoanalyser (Instrumentation Laboratory Inc.).

Urinary protein was measured using the modified Bradford assay (Bio-Rad Laboratories, Richmond, CA). Urinary thromboxane $\mathbf{B}_{2}$ (the stable metabolite of thromboxane $A_{2}$ ) was assayed in duplicate, respectively, by radioimmunoassay using antisera raised in rabbits using methodology modified from Reingold et al. (25). Cross-reactivities with other prostaglandins at $50 \%$ displacement were $<0.025 \%$ for the thromboxane $B_{2}$ antiserum.

Statistics. Results are expressed as mean \pm SEM. For comparison between control and EFAD groups of animals an unpaired Student's $t$ test was used. For comparisons between the same animals studied at different time points a paired Student's $t$ test was used. $P<0.05$ was considered significant.

\section{Results}

Validation of EFAD. The rats fed the diet deficient in essential fatty acids exhibited the characteristic dermatitis and on average weighed $10 \%$ less than age matched animals fed the control $\operatorname{diet}(222.7 \pm 7.98$ versus $195.1 \pm 4.46 \mathrm{~g} ; P<0.005)$.

The fatty acid analysis of the livers from animals raised on the diet deficient in essential fatty acids revealed a decrease in (n-6) fatty acids and the accumulation of (n-9) fatty acids, particularly 20:3 (n-9) (Mead acid) which is not present normally. The 20:3(n-9) to arachidonate ratio in the liver typically ranged from 1.8 to 3.8 , thus exceeding the minimal biochemical criteria for essential fatty acid deficiency which is a ratio of $>0.4$ (26).

Functional effects of PAN. $7 \mathrm{~d}$ after the administration of PAN both the EFAD and control rats developed the nephrotic syndrome (Table I), as evidenced by the development of heavy proteinuria, hypercholesterolemia, and hypoalbuminemia. The degree of proteinuria tended to be less in the EFAD rats, although it was only significantly different on day 21 after the administration of PAN. However, this was reflected in a higher plasma albumin in the EFAD rats. The severity of the nephrotic syndrome (as judged by the degree of proteinuria, hypoalbuminemia, and hypercholesterolemia) was greatest $14 \mathrm{~d}$ after the administration of PAN in both groups, and tended to resolve over the following $14 \mathrm{~d}$ (plasma albumin returned to control values, and proteinuria and hypercholesterolemia returned towards baseline). Interestingly, despite a higher plasma albumin after the induction of the nephrotic syndrome, the EFAD rats developed comparable degrees of hypercholesterolemia as controls; indeed, the cholesterol was significantly greater at day 14 in EFÁD rats. They did not, however, develop significant hypertriglyceridemia.

The effect of the administration of PAN on BUN and plasma creatinine for both EFAD and control animals is shown in Fig. 1. The BUN and plasma creatinine were elevated compared to baseline values $7 \mathrm{~d}$ after the administration of PAN in both control and EFAD animals $(P<0.002$ and $P$ $<0.05$; day 7 versus day 0 for creatinine in control versus

Table I. The Effect of the Administration of PAN to Control (C) and EFAD Rats (E Rats)

\begin{tabular}{|c|c|c|c|c|c|}
\hline Days post pan & 0 & 7 & 14 & 21 & 28 \\
\hline \multicolumn{6}{|c|}{ Proteinuria $(m g / 24 h)$} \\
\hline $\mathrm{C}$ & $2.0 \pm 0.2$ & $257.7 \pm 47.7$ & $300.9 \pm 34.9$ & $223.3 \pm 58.3$ & $17.7 \pm 5.0$ \\
\hline $\mathrm{E}$ & $1.5 \pm 0.2$ & $149.2 \pm 45.1$ & $221.5 \pm 43.8$ & $83.1 \pm 14.1^{*}$ & $21.3 \pm 8.9$ \\
\hline \multicolumn{6}{|l|}{ Albumin $(g / d l)$} \\
\hline $\mathrm{C}$ & $3.8 \pm 0.1$ & $1.8 \pm 0.1$ & $1.9 \pm 0.1$ & $2.6 \pm 0.1$ & $3.3 \pm 0.1$ \\
\hline $\mathbf{E}$ & $3.9 \pm 0.1$ & $2.5 \pm 0.2^{*}$ & $2.6 \pm 0.1^{\ddagger}$ & $3.3 \pm 0.2^{*}$ & $4.0 \pm 0.0^{\ddagger}$ \\
\hline \multicolumn{6}{|l|}{ Cholesterol $(m g / d l)$} \\
\hline $\mathrm{C}$ & $49.0 \pm 3.9$ & $159.4 \pm 15.8$ & $261.0 \pm 34.3$ & $256.9 \pm 52.7$ & $109.9 \pm 20.0$ \\
\hline $\mathbf{E}$ & $46.7 \pm 3.8$ & $125.2 \pm 20.8$ & $400.8 \pm 29.0^{*}$ & $216.5 \pm 17.8$ & $94.5 \pm 4.1$ \\
\hline \multicolumn{6}{|c|}{ Triglyceride $(m g / d l)$} \\
\hline C & $53.7 \pm 5.7$ & $99.9 \pm 2.9$ & $688.0 \pm 205.2$ & $336.6 \pm 52.6$ & $74.6 \pm 12.3$ \\
\hline $\mathbf{E}$ & $58.8 \pm 3.6$ & $79.6 \pm 13.9$ & $173.5 \pm 41.7^{*}$ & $70.3 \pm 6.9^{\ddagger}$ & $48.9 \pm 4.1$ \\
\hline
\end{tabular}

Serum parameters were measured on tail vein blood samples at $0,7,14,21$, and $28 \mathrm{~d}$ after the administration of PAN $15 \mathrm{mg} / 100 \mathrm{~g} \mathrm{BW}$. Both control and EFAD rats developed significant proteinuria, hypoalbuminemia, and hypercholesterolemia, which resolved over $28 \mathrm{~d}$. $* P<0.05$ control versus EFAD; $\quad{ }^{\ddagger} P<0.002$ control versus EFAD. 

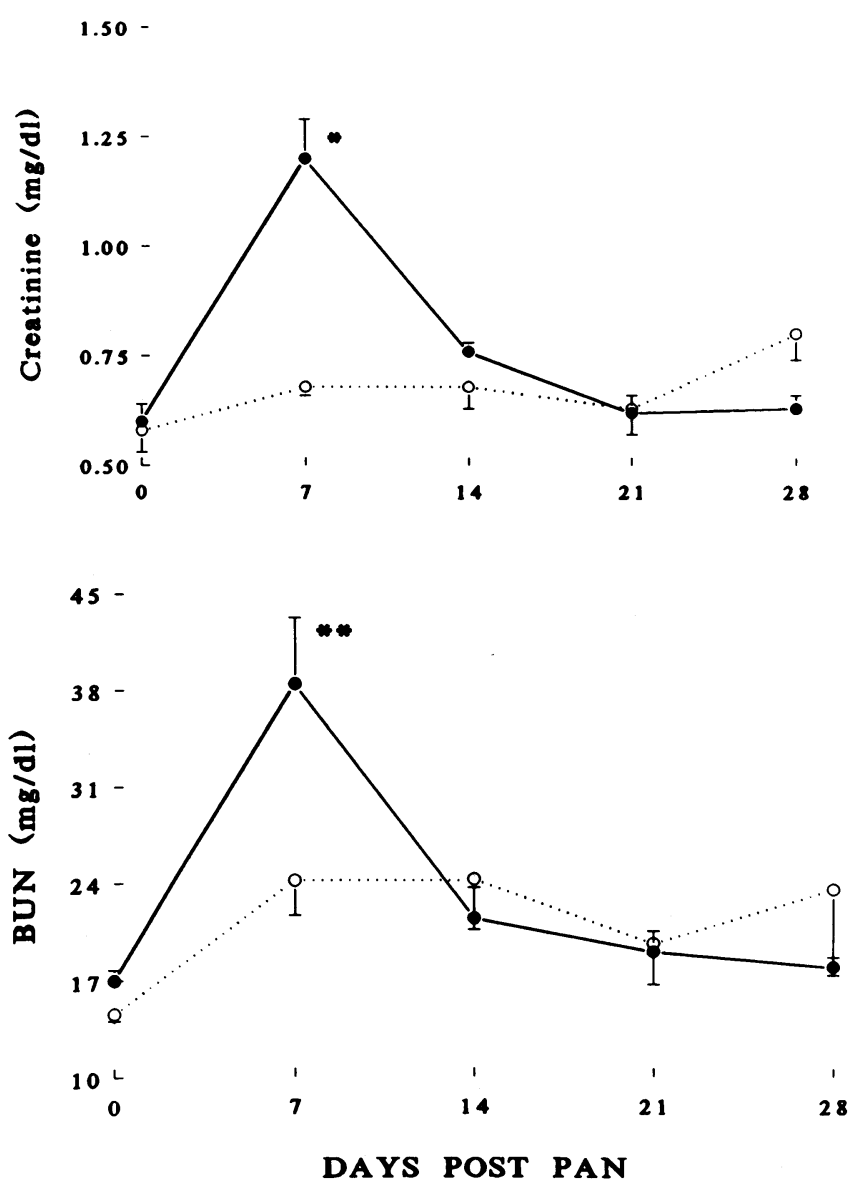

Figure 1. The effect of PAN on BUN and creatinine. BUN and creatinine were measured in tail vein blood samples from rats fed a control diet $(\bullet)$ and rats fed a diet deficient in essential fatty acids $(0)$ before $(0)$, and 7, 14, 21, and $28 \mathrm{~d}$ after the administration of PAN 15 $\mathrm{mg} / 100 \mathrm{~g} \mathrm{BW}$ i.p. At day 7, BUN and creatinine were significantly higher in control than in EFAD rats $\left({ }^{*} P<0.001\right.$ and ${ }^{* *} P<0.05$ for control versus EFAD.)

EFAD, respectively; and $P<0.02$ day 7 versus day 0 for BUN in both control and EFAD). However, the rise in BUN and plasma creatinine was minimal in the EFAD animals and was significantly less than in control animals $(24.2 \pm 2.5$ versus $38.5 \pm 4.8 \mathrm{mg} / \mathrm{dl}, P<0.05$; and $0.68 \pm 0.02$ versus $1.20 \pm 0.09$ $\mathrm{mg} / \mathrm{dl}, P<0.001 ;$ EFAD versus control for BUN and plasma creatinine at day 7 , respectively). The plasma creatinine had returned to a value not significantly different from baseline by day 14 in the EFAD rats and by day 21 in control rats.

Urinary excretion rates of thromboxane $B_{2}$ were significantly greater under basal conditions in animals fed the control diet than in animals fed the diet deficient in essential fatty acids (Fig. 2). After the induction of the nephrotic syndrome, there was a fall in thromboxane $B_{2}$ excretion in animals fed the control diet, such that the excretion rate was not different from that in the EFAD animals at $7 \mathrm{~d}$ after the administration of PAN. The excretion of thromboxane $B_{2}$ increased towards baseline as the nephrotic syndrome resolved. The same pattern was seen when urinary thromboxane was factored for urinary creatinine (data not shown).

The results of the inulin and PAH clearance studies in control and EFAD rats 0 (noninjected), 4 , and $7 \mathrm{~d}$ after the

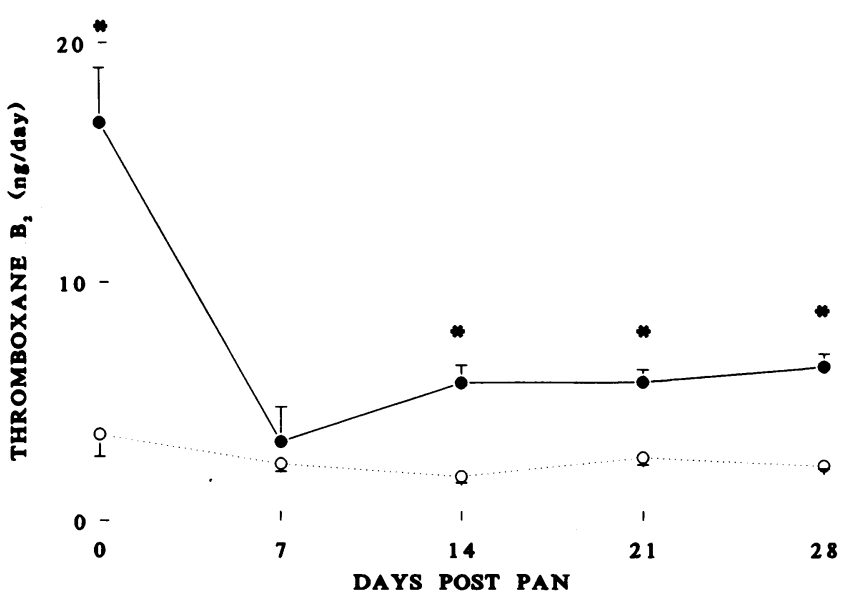

Figure 2. The effect of PAN on urinary thromboxane $\mathrm{B}_{2}$ excretion. Thromboxane $B_{2}$ excretion was measured before (0), and 7, 14, 21, and $28 \mathrm{~d}$ after the administration of PAN to rats fed a control diet $(\bullet)$ and rats fed a diet deficient in essential fatty acids $(0)$. Thromboxane $B_{2}$ was analyzed as outlined in the methods section. $\left({ }^{*} P\right.$ $<0.002$ for control versus EFAD.)

injection of PAN are shown in Fig. 3. As can be seen $4 \mathrm{~d}$ after the injection of PAN, the inulin clearance $\left(\mathrm{C}_{\text {in }}\right)$ of control rats was significantly lower $(50 \%)$ than in noninjected rats. There was a further fall in $C_{\text {in }}$ in the control rats by day 7 to $<5 \%$ of values obtained in noninjected rats. At both 4 and $7 \mathrm{~d}$ after the injection of PAN the EFAD rats had a markedly higher $C_{\text {in }}$ than control rats $(8.43 \pm 1.27$ versus $4.99 \pm 0.59 \mathrm{ml} / \mathrm{min} / \mathrm{kg} \mathrm{BW}$, $P<0.05$; and $5.21 \pm 1.19$ versus $0.39 \pm 0.08 \mathrm{ml} / \mathrm{min} / \mathrm{kg} \mathrm{BW}, P$ $<0.002$ at 4 and $7 \mathrm{~d}$, respectively). However, there was still a fall in the $C_{\text {in }}$ in EFAD rats, and the value at $7 \mathrm{~d}$ after the administration of PAN was significantly different from rats not given PAN $(5.21 \pm 1.19$ versus $11.94 \pm 0.71, P<0.001)$. In control rats $7 \mathrm{~d}$ after the injection of PAN, the clearance of PAH $\left(C_{\mathrm{PAH}}\right)$ had fallen dramatically to $<10 \mathrm{ml} / \mathrm{min} / \mathrm{kg} \mathrm{BW}$. In contrast, in EFAD rats, $\mathrm{C}_{\mathrm{PAH}}$ was maintained at levels similar to that in noninjected rats at both 4 and $7 \mathrm{~d}$ after the injection of PAN.

There was avid sodium retention in the control animals with the development of the nephrotic syndrome. Sodium excretion fell from $3.63 \pm 1.67 \mu \mathrm{mol} / \mathrm{min}$ in the animals that had not received PAN to $0.49 \pm 0.14 \mu \mathrm{mol} / \mathrm{min}$ at day $4(P<0.01)$ and to $0.143 \pm 0.025 \mu \mathrm{mol} / \mathrm{min}$ at day $7(P<0.002)$.

Analysis of the plasma and urine of the animals that underwent clearance studies confirmed the data obtained in rats studied longitudinally; namely a significantly higher creatinine in the control group at day $7(1.7 \pm 0.18$ versus $0.55 \pm 0.07$ $\mathrm{mg} / \mathrm{dl}, P<0.001)$, and a tendency for greater proteinuria in the control animals $(28.5 \pm 5.6$ versus $9.3 \pm 2.2 \mathrm{mg} / 24 \mathrm{~h}, P$ $<0.05$ at day 4 ; and $420.7 \pm 73.2$ versus $197.3 \pm 26.2 \mathrm{mg} / 24 \mathrm{~h}$, $P<0.05$ at day 7) and a better maintained plasma albumin.

Interstitial and glomerular macrophage quantification. The results of the quantification of the macrophage infiltrate is shown in Fig. 4. There were significantly more macrophages per high power field in the kidneys from control animals $7 \mathrm{~d}$ after the administration of PAN than in the kidneys from EFAD animals. Indeed, the macrophage number in the kidneys of two of the EFAD animals at day 7 was similar to the background. The macrophages in the kidneys of rats fed a standard diet and given PAN were located predominantly 

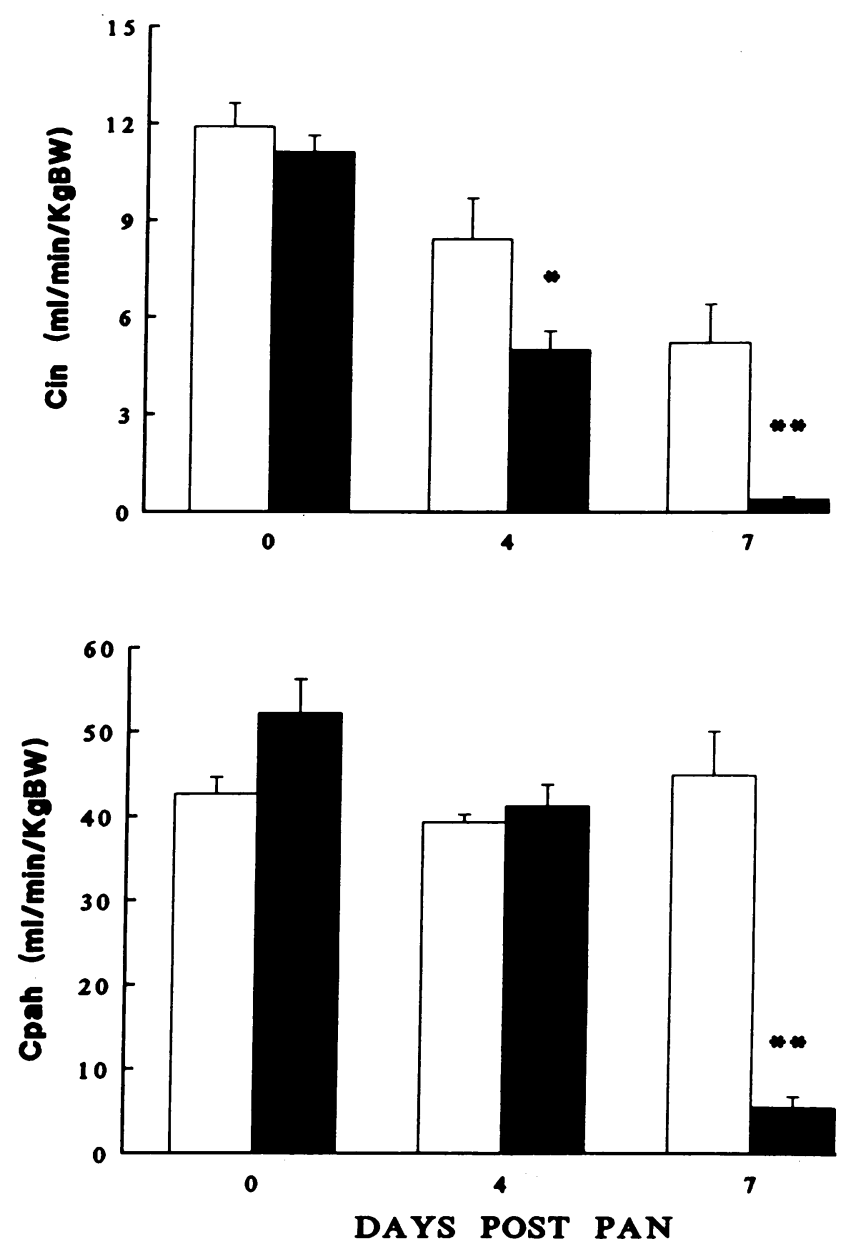

Figure 3. The effect of PAN on inulin and PAH clearances. $C_{i n}$ and $\mathrm{C}_{\mathrm{PAH}}$ were measured in rats fed a control diet $(\square)$ and rats fed a diet deficient in essential fatty acids (ㅁ) 0 (preexposure), 4 and $7 \mathrm{~d}$ after the administration of PAN. The rats were pair fed before the administration of the PAN. $C_{\text {in }}$ fell in both control and EFAD rats but was significantly higher in EFAD rats 4 and $7 \mathrm{~d}$ after the administration of PAN. $C_{\text {PAH }}$ did not fall in response to PAN in EFAD rats, but did fall in rats raised on a control diet. $\left({ }^{*} P<0.05\right.$ and ${ }^{* *} P<0.002$ for control versus EFAD).

around the glomerular stalk and proximal tubule. A representative section of a kidney from a control animal at day 7 is shown in Fig. 5.

There was no change in the number of glomerular macrophages $7 \mathrm{~d}$ after the administration of PAN in rats fed a normal diet $(9.0 \pm 0.5$ macrophages/glomerulus in PAN-injected animals and $8.6 \pm 0.5$ in noninjected controls).

Glomerular eicosanoid production. The effects of PAN on the production of thromboxane $B_{2}$ generation by isolated glomeruli is shown in Fig. 6. PAN did not increase either basal or ionomycin-stimulated thromboxane $\mathbf{B}_{2}$ production. Neither did PAN affect prostaglandin $\mathrm{E}_{2}$ or leukotriene $\mathrm{B}_{4}$ production by isolated glomeruli (data not shown).

Effects of thromboxane synthesis inhibition. The results of inhibiting the generation of the vasoconstrictor thromboxane $A_{2}$ are shown in Fig. $7.7 \mathrm{~d}$ after the administration of PAN, $C_{\text {in }}$ was severely reduced in animals fed the control diet. However, the administration of the thromboxane synthase inhibitor OKY-046 did not result in a higher $C_{\text {in }}$ than in concurrently

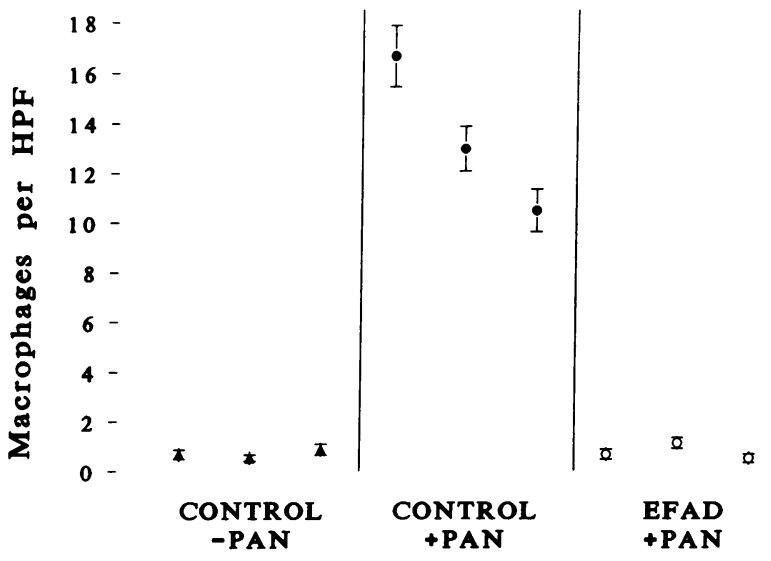

Figure 4. Quantification of the interstitial infiltrate following PAN. Macrophage number in the interstitium was quantified in nonnephrotic rats and $7 \mathrm{~d}$ after the administration of PAN. ( $\Delta$ ) Rats on a control diet not given PAN; (๑) rats on a control diet given PAN $7 \mathrm{~d}$ previously; $(O)$ rats on a diet deficient in essential fatty acids given PAN $7 \mathrm{~d}$ earlier. Frozen sections of kidney were stained for macrophages using an ED1 antibody as described in the text and a mean number of macrophages in 15 high-powered fields taken.

studied controls. Neither did the administration of OKY-046 affect the level of proteinuria $7 \mathrm{~d}$ after the administration of PAN (194.6 \pm 24.7 versus $211.18 \pm 41.94$ with and without OKY-046, respectively).

Effects of leukopenia on renal function. Essential fatty acid deficiency may ameliorate renal dysfunction in this model of nephrosis by preventing leukocyte infiltration, by inhibiting a vasoactive fatty acid metabolite other than thromboxane $A_{2}$, or by a combination of the two. To delineate the contribution of infiltrating leukocytes, a group of rats on a control diet ( $n$ $=5$ ), received lethal irradiation $3 \mathrm{~d}$ before being studied on day 7 after the administration of PAN. The kidneys were shielded to prevent direct radiation toxicity. This protocol depletes the kidneys of resident leukocytes and induces monocyte and neutrophil depletion within $24 \mathrm{~h}$ (15). Two control groups were analyzed with respect to interstitial leukocyte content, one group on a standard diet $(n=6)$, and one group on a standard diet that received PAN $7 \mathrm{~d}$ before study $(n=6)$. As shown in Fig. 8, PAN-induced nephrosis was associated with a $>10$-fold increase in interstitial leukocytes on day 7 . The irradiated, nephrotic rats demonstrated virtually complete depletion of interstitial leukocytes.

The two groups of nephrotic animals, irradiated and nonirradiated, were compared with respect to several parameters of renal dysfunction. We have previously shown that this irradiation protocol has no effect on renal function in nonnephrotic rats on standard diets (16). As seen in Table II, both groups demonstrated comparable proteinuria. The $\mathrm{x}$-irradiated group experienced a significant loss of body weight, $\sim 13 \%$. Hematocrit was the same for both groups. BUN was significantly lower for the irradiated group, comparable to that seen in EFAD nephrosis (Fig. 1). Most significantly, despite the same degree of proteinuria, the leukopenic nephrotic animals manifested almost a tripling of the inulin clearance and a greater than twofold increase in PAH clearance. Thus, the prevention of leukocyte influx into the renal interstitium appears to account for $60-80 \%$ of the ameliorating effects of 

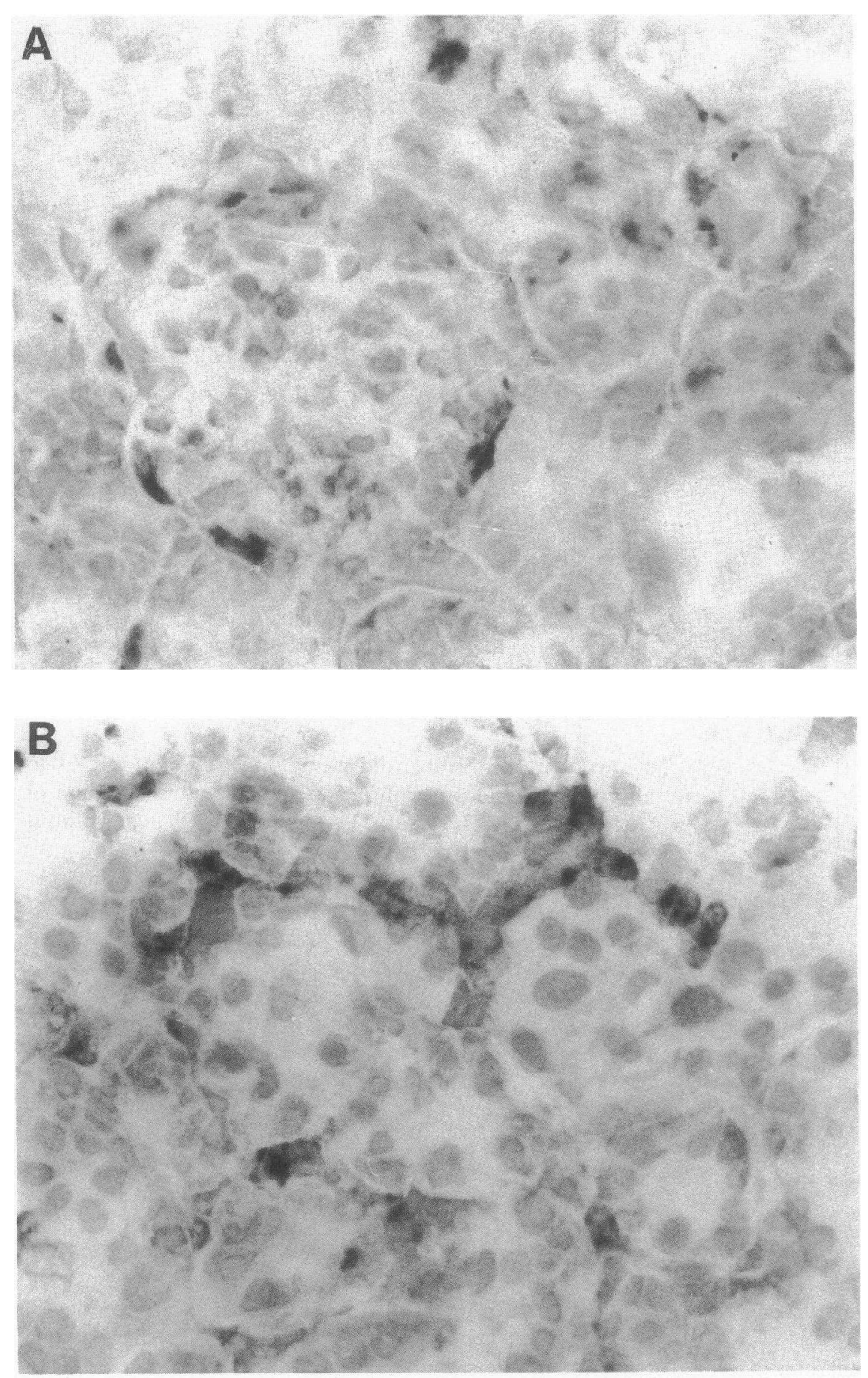

Figure 5. A representative photomicrograph of a section from a kidney of a rat fed a control diet $7 \mathrm{~d}$ after the administration of PAN. A frozen section of the kidney was stained for macrophages using an ED1 antibody as described in the text. The macrophages are seen predominantly in the periglomerular region and around the proximal tubules of the kidney.
EFAD on the impaired renal function in this animal model of the nephrotic syndrome.

\section{Discussion}

Transient exposure of the kidney to PAN induces acute toxicity in the renal glomerular epithelium causing a decrease in the negative charge of the glomerular capillary wall and an increase in the passage of polyanion molecules such as albumin, resulting in the development of nephrosis $(5,27,28)$. Concomitantly, there is avid sodium retention and a decrease in glomerular filtration rate secondary to a fall in the ultrafiltration coefficient and plasma flow per nephron $(5,6)$. Recently, it has become apparent that an interstitial infiltrate develops after administration of PAN (2).

The results presented in this paper demonstrate that the interstitial leukocyte infiltrate that occurs after the administration of PAN is causally linked with the severe reduction in glomerular filtration rate and renal blood flow at $7 \mathrm{~d}$. At this time, the infiltrate is only in the interstitium, with no change in glomerular macrophage number.

EFAD has previously been shown to be protective against the lethal glomerulonephritis that develops in murine lupus (8). Rats raised on this diet have a reduced number of glomerular and interstitial macrophages (10). EFAD is associated with a marked inhibition of monocyte migration into the glo- 


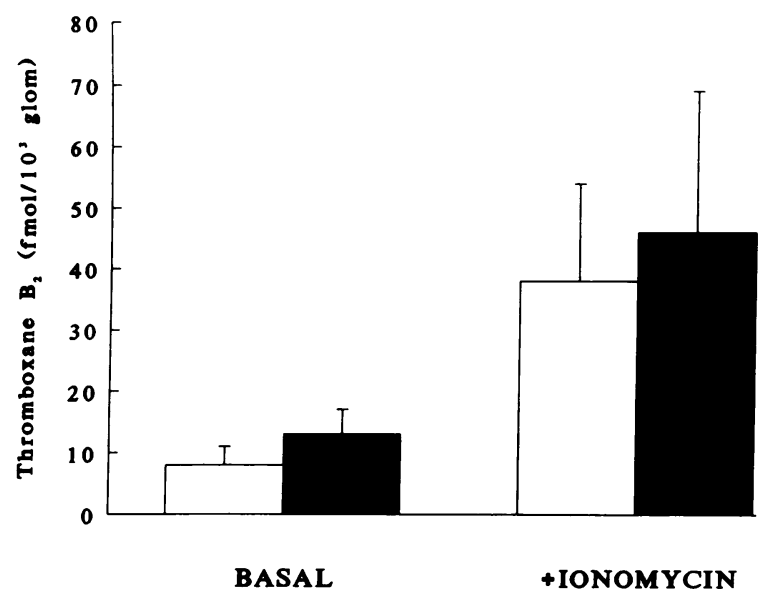

Figure 6. The effect of PAN on glomerular thromboxane $\mathrm{B}_{2}$ production. Glomerular $\mathrm{TxB}_{2}$ synthesis was assayed in nonnephrotic animals fed a control diet $(\square)$ and in animals $7 \mathrm{~d}$ after the administration of PAN (a). Glomeruli from nonnephrotic and nephrotic animals were isolated and incubated as detailed in Methods.

Glomerular $\mathrm{TxB}_{2}$ synthesis was determined by specific radioimmunoassay and normalized for the number of glomeruli in the incubation. Constitutive (basal) and ionomycin-stimulated production are shown. There was no significant change in either basal or ionomycin-stimulated glomerular thromboxane $B_{2}$ production $7 \mathrm{~d}$ after PAN administration.

merulus after the administration of nephrotoxic serum or aggregated protein $(11,12)$. In contrast, inflammation-induced glomerular infiltration by polymorphonuclear leukocytes is not affected by EFAD (12).

This study demonstrates for the first time that animals raised on an EFAD diet are unable to develop an interstitial infiltrate of macrophages in response to PAN. The mechanism whereby inflammatory stimuli fail to result in the recruitment of macrophages in EFAD remains to be defined, but the failure of the kidney to produce a chemoattractant, a failure of the macrophages to respond, or both are possible explanations.

Rats raised on a EFAD diet were also protected against the

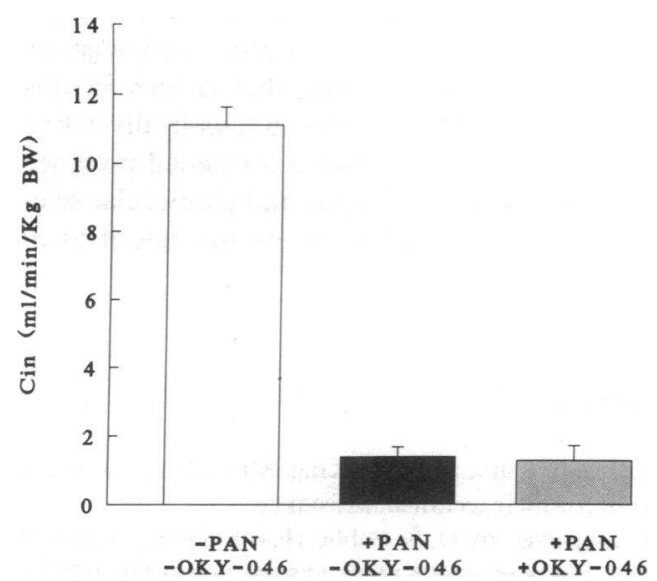

Figure 7. The effect of the administration of OKY-046 on the development of renal dysfunction after PAN. Rats fed a control diet were studied 7 days following the administration of PAN. OKY-046 20 $\mathrm{mg} / \mathrm{kg}$ BW or saline was given subcutaneously twice a day following the administration of PAN. PAN induced a severe fall in $\mathrm{C}_{\text {in }}$ that was not ameliorated by OKY-046. The $C_{\text {in }}$ values for rats not given PAN are also shown (-PAN, and -OKY-046).

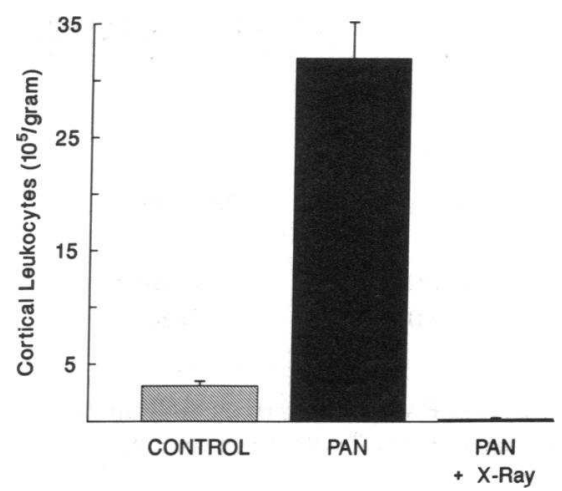

Figure 8. The effect of bone marrow irradiation on the renal cortical leukocyte content of nephrotic rats. Rats, on a standard diet, were given PAN and their kidneys analyzed for the presence of leukocytes $7 \mathrm{~d}$ later. One group received systemic irradiation, with shielding of the kidneys, on day 4 after the administration of PAN; the kidneys were analyzed on day 7 . Analyses are presented as $10^{5}$ leukocytes/ gram wet weight of renal cortex. Results are means $(n=6$, controls; $n=6$ for PAN only; $n=5$ for PAN + irradiation) \pm SE.

severe decline in renal function that is seen after administration of PAN. Inulin clearance fell to $\sim 3.5 \%$ of preadministration values by day 7 in rats fed a standard chow. The inulin clearance also fell significantly in animals raised on the EFAD diet given PAN, but to only $50 \%$ of preexposure values. This resulted in an inulin clearance which was 13 times that in the animals fed the standard chow. This preservation in renal function is associated with the reduction in the interstitial infiltration by macrophages in the EFAD animals. The beneficial effects on renal function observed in nephrotic rats fed a standard diet and made leukopenic by irradiation proves that the predominant factor in the protection conferred by EFAD is its inhibition of macrophage infiltration. This is the first demonstration that the proteinuria-induced influx of macrophages into the renal interstitium directly modulates renal blood flow and glomerular filtration and, in turn, is modulated by manipulation of dietary lipids.

Recently, Diamond et al. (29) demonstrated that inducing EFAD in rats during the transient phase of PAN-induced nephrosis dramatically inhibited the glomerular sclerosis and interstitial fibrosis typically observed in the kidneys 4-6 mo after a single dose of PAN (29). Noteworthy was the fact that the rats were essential fatty acid deficient only during the first month of proteinuria; they were placed on a standard diet thereafter. That study did not evaluate renal function or the interstitial infiltrate in the acute phase of the disease. We would propose that EFAD inhibits the acute renal ischemia of this model of nephrosis by preventing the interstitial leukocyte infiltration. Subsequent protection from interstitial fibrosis in the chronic phase of proteinuria may be due either to maintenance of renal blood flow in the acute phase or to sustained depletion from the interstitium and glomeruli of macrophages, which may promote nephrosclerosis via their established capacity to degrade extracellular matrix, stimulate fibroblast proliferation, and promote collagen production, to cite just a few of their chronic inflammatory properties (30).

It is of note that the inulin clearance in the EFAD animals was still reduced to $50 \%$ of preexposure values. This is consistent with PAN having a direct toxic effect on the glomerular epithelium $(31,32)$. Such an effect could directly modify the filtration characteristics of the glomerulus and result in a de- 
Table II. The Effect of X-Irradiation on Renal Function in PAH-induced Nephrosis

\begin{tabular}{|c|c|c|c|c|c|c|}
\hline & BW & Hct & BUN & Proteinuria & $C_{\text {inulin }}$ & $\mathrm{C}_{\mathrm{PAH}}$ \\
\hline & $g$ & $\%$ & $m g / d l$ & $g / 24 h$ & $\mathrm{ml} / \mathrm{min} / \mathrm{kg}$ & $\mathrm{ml} / \mathrm{min} / \mathrm{kg}$ \\
\hline $\operatorname{PAN}(n=6)$ & $237.5 \pm 7.8$ & $36.78 \pm 1.8$ & $49.0 \pm 3.8$ & $91.2 \pm 9.2$ & $1.04 \pm .18$ & $13.91 \pm 2.67$ \\
\hline \multicolumn{7}{|c|}{ PAN $+x$-irradiation } \\
\hline$(n=5)$ & $207.9 \pm 2.9$ & $36.8 \pm 0.5$ & $22.2 \pm 2.1$ & $114.7 \pm 27.8$ & $2.98 \pm .26$ & $34.33 \pm 5.88$ \\
\hline$p$ & $<0.025$ & N.S. & $<0.001$ & N.S. & $<0.001$ & $<0.01$ \\
\hline
\end{tabular}

Age-matched female rats received PAN ( $15 \mathrm{mg} / 10 \mu \mathrm{g} \mathrm{BW}$ ) on day 0 . One group received 1,315 rads on day 4, with lead shielding of the kidneys. All animals were sacrificed on day 7 with serum parameters measured on tail vein samples before determination of inulin and PAH clearances. Values, means \pm SE.

crease in inulin clearance, and would not be modified by the EFAD state. Strikingly, renal plasma flow as assessed by the clearance of PAH was completely preserved in the EFAD animals, where, as in control animals, it had fallen dramatically 7 $\mathrm{d}$ after the exposure to PAN.

Although the leukocyte infiltrate appears to account for $60-80 \%$ of the impaired renal function in this model, the remainder should perhaps be attributable to impaired synthesis of a vasoactive eicosanoid. In EFAD, the production of both cyclooxygenase and lipoxygenase products, particularly thromboxane $A_{2}$, is impaired both basally and in acute glomerulonephritis $(10,12)$. Thromboxane $A_{2}$ is the principal vasoconstrictive eicosanoid implicated in the pathogenesis of several forms of acute renal failure, including obstructive nephropathy $(16,19,33)$ in which it is evident that infiltrating cells are a major source of the enhanced thromboxane $A_{2}$ production $(16,19)$, glycerol-induced renal failure $(34,35)$, and ischemia (36). Furthermore, in adriamycin-induced nephrosis there is an enhanced urinary excretion rate of thromboxane $B_{2}$ and enhanced glomerular production of thromboxane $B_{2}$. Inhibition of thromboxane synthesis significantly reduces proteinuria (13). The source of the enhanced thromboxane production in this model is thought to be the glomerular epithelium, since no leukocyte infiltrate has been observed in the kidney after administration of adriamycin $(13,37)$. Our data on the effects of leukopenia and on inhibition of leukocyte influx may thus explain why adriamycin nephrosis differs from PAN nephrosis in its lack of correlative glomerular dysfunction.

Before the administration of PAN, thromboxane $\mathrm{B}_{2}$ excretion was significantly less in the EFAD animals than those on the control diet, consistent with EFAD animals being unable to synthesize thromboxane $A_{2}$. However, after exposure to PAN, thromboxane $B_{2}$ excretion in control animals did not increase. Rather, the excretion of thromboxane $B_{2}$ fell and increased again only as the nephrosis resolved. In addition, glomeruli isolated $7 \mathrm{~d}$ after the administration of PAN, from rats fed the control diet, did not show enhanced basal or ionomycin-stimulated production of thromboxane $B_{2}$ or leukotriene $B_{4}$ over nonnephrotic rats. Furthermore, administration of the thromboxane synthase inhibitor OKY-046, at doses that have previously been shown to be highly effective in suppressing thromboxane $B_{2}$ excretion (38), did not prevent the acute fall in glomerular filtration rate at $7 \mathrm{~d}$, nor did it ameliorate the proteinuria. These observations would suggest that thromboxane $A_{2}$, whether derived from intrinsic renal cells or infiltrating macrophages, is not important in the pathogenesis of the decline in renal function after the administration of PAN. It remains possible that an as yet unidentified metabolite of linoleate or arachidonate contributes in part to the decline in renal function observed in this model and that its synthesis is impaired by EFAD. Clarification of this possibility must await further investigation.

The potential role of other known humoral mediators of renal vasoconstriction in acute, leukocyte-dependent depression of renal blood flow remains to be clarified. It is known that administration of angiotensin 1 converting enzyme inhibitor does not reverse the decreased glomercular filtration rate in the acute phase of PAN nephrosis, the phase that we have studied (39), although it does normalize glomerular filtration in the chronic phase of the model, 70 wk after PAN administration, characterized by interstitial fibrosis and glomerular sclerosis in the untreated animal. There is no information on a role for endothelin in this model of renal ischemia, nor is it known whether endothelin release is subject to modulation by lipids or by the presence of activated leukocytes.

In summary, we have shown that there is a significant influx of macrophages into the interstitium $7 \mathrm{~d}$ after the administration of PAN, at a time when glomerular macrophage number is unaltered. This is associated with a decline in inulin and PAH clearances at this time, which is not related to the production of thromboxane $A_{2}$. EFAD effectively prevents the recruitment of macrophages into the interstitium. At the same time, the fall in inulin clearance is ameliorated and the fall in PAH clearances is prevented by the EFAD diet. Proteinuria and hypercholesterolemia, however, still occur. This suggests that the acute decline in renal function that is seen in this model is modulated via the infiltration of metabolically active macrophages into the interstitium, whose continued presence may contribute to the interstitial fibrosis and glomerular sclerosis that are the distant sequelae to the events described in these studies.

\section{Acknowledgments}

The authors would like to thank Mrs. Sue King, Mrs. Claire Pederson, and Mr. Dan Martin for their technical assistance.

This work was supported by U. S. Public Health Service National Institute of Diabetes, Digestive and Kidney Disease grants DK-09976, DK-07126, AM-36277, DK 40321, DK 37879, and HL 01313. Additional support was provided by the Mallinckrodt Foundation and by the Communities Foundation of Texas. Dr. Kevin Harris is a recipient of a Research Fellowship from the National Kidney Foundation. Dr. George F. Schreiner is an Established Investigator of the American Heart Association. Dr. James B. Lefkowith is the recipient of an International Life Sciences Institute Award. 


\section{References}

1. Frenk, S., I. Antonowicz, J. M. Craig, and J. Metcoff. 1955. Experimental nephrotic syndrome induced in rats by aminonucleoside. Renal lesions and body electrolyte composition. Proc. Soc. Exp. Biol. Med. 89:424-427.

2. Eddy, A. A., and A. F. Michael. 1988. Acute tubulointerstitial nephritis associated with aminonucleoside nephrosis. Kidney Int. 33:14-23.

3. Diamond, J. R., and M. J. Karnovsky. 1986. Focal and segmental glomerulosclerosis following a single intravenous dose of puromycin aminonucleoside. Am. J. Pathol. 122:481-487.

4. Ryan, G. B., and M. J. Karnovsky. 1975. An ultrastructural study of the mechanisms of proteinuria in aminonucleoside nephrosis. Kidney Int. 8:219-232.

5. Bohrer, M. P., C. Baylis, C. R. Robertson, and B. M. Brenner. 1977. Mechanisms in the puromycin-induced defects in the transglomerular passage of water and macromolecules. J. Clin. Invest. 60:152-161.

6. Ichikawa, I., H. G. Rennke, J. R. Hoyer, K. F. Badr, N. Schor, J. L. Troy, C. P. Lechene, and B. M. Brenner. 1983. Role of intrarenal mechanisms in the impaired salt excretion of experimental nephrotic syndrome. J. Clin. Invest. 71:91-103.

7. Schreiner, G. F., R. S. Cotran, and E. R. Unanue. 1984. Modulation of Ia and leukocyte common antigen expression in rat glomeruli during the course of glomerulonephritis and aminonucleoside nephrosis. Lab. Invest. 51:524-533.

8. Hurd, E. R., J. M. Johnston, J. R. Okita, P. C. MacDonald, M. Ziff, and J. N. Gilliam. 1981. Prevention of glomerulonephritis and prolonged survival in New Zealand Black/New Zealand White $F_{1}$ hybrid mice fed an essential fatty acid-deficient diet. J. Clin. Invest. 67:476-485.

9. Kelley, V. E., A. Ferretti, S. Izui, and T. B. Strom. 1985. A fish oil diet rich in eicosopentanoic acid reduces cyclooxygenase metabolites and suppresses lupus in MRL-1 pr mice. J. Immunol. 134:19141919.

10. Lefkowith, J. B., and G. Schreiner. 1987. Essential fatty acid deficiency depletes rat glomeruli of resident macrophages and inhibits angiotensin II-induced eicosanoid synthesis. J. Clin. Invest. 80:947956.

11. Lefkowith, J. B., B. Rovin, and G. Schreiner. 1989. Depletion of resident glomerular macrophages by essential fatty acid deficiency protects against glomerulonephritis. In Advances in Prostaglandin, Thromboxane, and Leukotriene Research. B. Samuelson, Y. K. Wong, F. F. Sun, editors. Raven Press, New York. 560-563.

12. Schreiner, G. F., B. Rovin, and J. Lefkowith. 1989. The anti-inflammatory effects of essential fatty acid deficiency in experimental glomerulonephritis. J. Immunol. 143:3192-3199.

13. Remuzzi, G., L. Imberti, M. Rossini, C. Morelli, G. Carminati, G. M. Cattaneo, and T. Bertani. 1985. Increased glomerular thromboxane synthesis as a possible cause for proteinuria in experimental nephrosis. J. Clin. Invest. 75:94-101.

14. Purkerson, M. L., and S. Klahr. 1989. Prior inhibition of vasoconstrictors normalizes GFR in post-obstructed kidneys. Kidney Int. 35:1306-1314.

15. Schreiner, G. F., and E. Unanue. 1984. Origin of the rat mesangial phagocyte and its expression of the leukocyte common antigen. Lab. Invest. 51:515-523.

16. Harris, K. P. G., G. F. Schreiner, and S. Klahr. 1989. Effect of leukocyte depletion on the function of the post-obstructed kidney in the rat. Kidney Int. 36:210-215.

17. Dijkstra, C., E. Dopp, P. Joling, and G. Kraal. 1985. The heterogeneity of mononuclear phagocytes in lymphoid organs: distinct macrophage subpopulations in the rt recognized by monoclonal antibodies EO1, EO2, and EO3. Immunology. 54:589-599.

18. Schreiner, G. F., J. Kiely, R. Cotran, and E. Unanue. 1981. Characterization of resident glomerular cells in the rat expressing Ia determinants and manifesting genetically restricted interactions with lymphocytes. J. Clin. Invest. 68:920-931.
19. Schreiner, G. F., K. P. G. Harris, M. L. Purkerson, and S. Klahr. 1988. Immunological aspects of acute ureteral obstruction: immune cell infiltrate in the kidney. Kidney Int. 34:487-493.

20. Lefkowith, J. B., B. A. Jakschik, P. Stahl, and P. Needleman. 1987. Metabolic and functional alterations in macrophages induced by essential fatty acid deficiency. J. Biol. Chem. 262:6668-6675.

21. Bligh, E. G., and W. Dyer. 1959. A rapid method of total lipid extraction and purification. Can. J. Biochem. Physiol. 37:911-917.

22. Lefkowith, J. B., V. Flippo, H. Sprecher, and P. Needleman. 1985. Paradoxical conservation of cardiac and renal arachidonate content in essential fatty acid deficiency. J. Biol. Chem. 260:1573615744.

23. Fuhr, J., J. Kaczmarczyk, and C. D. Kruttgen. 1955. Eine einfache colorimetrische Methode zur Inulinbestimmung fur Nierenclearanceuntersuchungen bei stoffwechselgesunden und Diabetikern. Klin. Wochenschr. 33:729-730.

24. Smith, H. W., N. Finkelstein, L. Aliminosa, B. Crawford, and M. Graber. 1945. The renal clearances of substituted hippuric acid derivatives and other aromatic acids in dog and man. J. Clin. Invest. 24:388-404.

25. Reingold, D. F., K. Waters, S. Holmberg, and P. Needleman. 1981. Differential biosynthesis of prostaglandins by hydronephrotic rabbit and cat kidneys. J. Pharmacol. Exp. Ther. 216:510-515.

26. Holman, R. 1960. The ratio of trienoic:tetraenoic acids in tissue lipids as a measure of essential fatty acid requirement. J. Nutrition. 70:405-410.

27. Michael, A. F., E. Blau, and R. L. Vernier. 1970. Glomerular polyanion alteration in aminonucleoside nephrosis. Lab. Invest. 23:649-657.

28. Olson, J. L., H. G. Rennke, and M. A. Venkatachalam. 1981. Alterations in the charge and size selectivity barrier of the glomerular filter in aminonucleoside nephrosis in rats. Lab. Invest. 44:271-279.

29. Diamond, J., I. Pesek, S. Ruggieri, and M. Karnovsky. 1989. Essential fatty acid deficiency during acute puromycin nephrosis ameliorates late renal injury. Am. J. Physiol. 257:F798-F807.

30. Nathan, C. 1987. Secretory products of macrophages. J. Clin. Invest. 79:319-326.

31. Caulfield, J. P., J. J. Reid, and M. J. Farquhar. 1976. Alterations of the glomerular epithelium in acute aminonucleoside nephrosis. Lab. Invest. 34:43-59.

32. Fishman, J. A., and M. J. Karnovsky. 1985. Effects of the aminonucleoside of puromycin on glomerular epithelial cells in vitro. Am. J. Pathol. 118:398-407.

33. Morrison, A. R., K. Nishikawa, and P. Needleman. 1977. Unmasking of thromboxane $A_{2}$ synthesis by ureter obstruction in the rabbit kidney. Nature (Lond.). 269:259-260.

34. Benabe, J. E., S. Klahr, M. K. Hoffman, and A. R. Morrison. 1980. Production of thromboxane $A_{2}$ by the kidney in glycerol-induced acute renal failure in the rabbit. Prostaglandins. 19:333-347.

35. Sraer, J. D., L. Moulonguet-Doleris, F. Delarue, J. Sraer, and R. Ardaillou. 1981. Prostaglandin synthesis by glomeruli isolated from rats with glycerol-induced acute renal failure. Circ. Res. 49:775-783.

36. Klausner, J. M., I. S. Paterson, L. Kobzik, C. Rodzen, C. R. Valeri, D. Shepro, and H. D. Hechtman. 1989. Vasodilating prostaglandins attenuate ischemic renal injury only if thromboxane is inhibited. Ann. Surg. 209:219-224.

37. Bertani, T., A. Poggi, R. Pozzoni, F. Delaini, G. Sacchi, Y. Thoua, G. Mecca, G. Remuzzi, and M. B. Donati. 1982. Adriamycin induced nephrotic syndrome in rats. Sequence of pathologic events. Lab. Invest. 46:16-23.

38. Purkerson, M. L., J. H. Joist, J. Yates, A. Valdes, A. Morrison, and S. Klahr. 1985. Inhibition of thromboxane synthesis ameliorates the progressive kidney disease of rats with subtotal renal ablation. Proc. Natl. Acad. Sci. 82:193-197.

39. Anderson, S., J. Diamond, M. Karnovsky, and B. Brenner. 1988. Mechanisms underlying transition from acute glomerular injury to late glomerular sclerosis in a rat model of nephrotic syndrome. $J$. Clin. Invest. 82:1757-1768. 